\title{
Anleitung zu wissenschaftlichen radiologischen Publikationen
}

\author{
Instructions for Original Radiological Research Manuscripts
}

Autoren

Institut
P. Bannas, G. Adam, T. A. Bley

Diagnostikzentrum, Klinik und Poliklinik für Diagnostische und Interventionelle Radiologie, Universitätsklinikum Hamburg Eppendorf, Hamburg
Key words

- technical aspects

- scientific writing

- radiological manuscript

- scientific radiological manuscript eingereicht 8.11 .2012

akzeptiert 14.1.2013

Bibliografie

DOI http://dx.doi.org/

10.1055/s-0032-1330747

Online-Publikation: 14.3.2013

Fortschr Röntgenstr 2013; 185:

533-538 @ Georg Thieme

Verlag KG Stuttgart · New York . ISSN 1438-9029

\section{Korrespondenzadresse}

\section{Dr. Peter Bannas}

Diagnostikzentrum, Klinik und Poliklinik für Diagnostische und Interventionelle Radiologie, Universitätsklinikum Hamburg Eppendorf

Martinistraße 52

20246 Hamburg

Tel.: ++ 49/40/741054023

Fax: ++49/40/74106799

P.bannas@uke.de

\section{Zusammenfassung \\ $\nabla$}

Ein Großteil aller zur Publikation eingereichter wissenschaftlicher Manuskripte beinhaltet formale und redaktionelle Fehler. Dadurch kommt es zur Ablehnung von wissenschaftlichen Daten in Journalen mit Peer-Review-Verfahren. Die vorliegende Arbeit soll jungen Wissenschaftlern eine Anleitung für die Erstellung eines geordneten und klar strukturierten radiologischen Manuskripts an die Hand geben.

\section{Einleitung}

Über das erfolgreiche Publizieren sind bereits zahlreiche Arbeiten, insbesondere im anglo-amerikanischen Sprachraum erschienen [1-18]. Ziel der vorliegenden Arbeit ist es, das Wichtigste aus diesen Publikationen den jungen Wissenschaftlern in der Radiologie im deutschsprachigen Raum zugänglich zu machen. Ermuntert durch die große Resonanz auf die Veranstaltung „Forschen und Publizieren“ während des Deutschen Röntgenkongresses 2012 in Hamburg möchten wir diesen Beitrag dem radiologischen Nachwuchs, den „hellsten Köpfen“ widmen.

Für das Schreiben eines wissenschaftlichen Manuskripts existieren klar definierte Regeln $[5,10]$. Diese Regeln werden jedoch häufig nicht eingehalten. Oft sind den Autoren diese Regeln nicht bewusst und/oder ein erfahrener Mentor, der auf diese hinweist, fehlt $[2,6]$. Es ist jedoch von großer Wichtigkeit, das Manuskript so zu organisieren, dass die Lesbarkeit und das Verständnis vereinfacht werden [7]. Es handelt sich nicht um vernachlässigbare Formfehler, die den Lesern Mühe bereiten, sondern vielmehr um Fehler, die weitreichende Konsequenzen für die Autoren nach sich ziehen: Das Manuskript wird erst nach wiederholten Anläufen, oder im schlimmsten Fall gar nicht, zur Publikation angenommen $[3,11]$.

\section{Abstract \\ $\nabla$}

Many scientific manuscripts submitted for publication are flawed by conceptual mistakes. This leads to the rejection of potentially valuable scientific data in renowned journals. This article intends to guide young researchers with respect to writing a well-structured scientific radiological manuscript.

Ein wissenschaftliches Manuskript sollte ein gut strukturierter Text sein, welcher die Forschungsergebnisse verständlich präsentiert. Das erhöht die Wahrscheinlichkeit erheblich, dass das Manuskript bei einem wissenschaftlichen Journal mit Gutachterverfahren zur Veröffentlichung angenommen wird. Natürlich wird vorausgesetzt, dass die zu präsentierenden Ergebnisse den Ansprüchen und dem Themenspektrum des Journals entsprechen. Um dies nachzuvollziehen, muss man die Entscheidungsprozesse verstehen, die bei der Begutachtung eines Manuskripts eine Rolle spielen [4]. Zunächst entscheidet der Herausgeber bei jedem eingereichten Manuskript, ob es zu dem Themenspektrum der Zeitschrift passt. An diesem Punkt kann ein Manuskript schon aufgrund einer falschen Thematik in der Überschrift ohne weitere Begutachtung abgelehnt werden. Bei den übrigen prinzipiell infrage kommenden Manuskripten spielt neben der Überschrift die Zusammenfassung, der „Abstract“, eine entscheidende Rolle [12]. Anschließend wird das Manuskript zur Begutachtung in der Regel an zwei Gutachter (,Reviewer“) geschickt, die sich mit der Thematik auskennen („Experts in the Field“) [14]. Es ist wichtig, dass der Inhalt schnell erfassbar und klar strukturiert im Manuskript präsentiert wurde - dies setzt eine möglichst standardisierte sowie fokussierte Struktur voraus 
$[4,12,13]$. Ist das Manuskript schlecht strukturiert und unverständlich, wird der negative Eindruck der Gutachter direkt an die Herausgeber weitergegeben [17]. Die meisten Journale haben Bewertungsskalen mit Unterkategorien, nach denen die Gutachter ein Manuskript zu benoten haben - neben einem Freitext mit spezifischen Kommentaren. Neben einer Benotung der Originalität (1), dem Innovationsgrad (2) und der Prüfung auf grobe Fehler (3) werden auch die Formalien benotet: Strukturierung des Textes (4), Anzahl und Qualität der Abbildungen (5), Sinnhaftigkeit der Schlussfolgerung (6) sowie nicht zuletzt Grammatik, Stil und Syntax (7). Aus diesen Unterkategorien wird eine Gesamtnote berechnet. Interessanterweise sind im vorgenannten Beispiel nur 3 der 7 Notenkategorien abhängig von der Qualität der eigentlichen Forschungsergebnisse, die übrigen 4 Kategorien betreffen die Formalien des eingereichten Manuskripts.

\section{Struktur eines wissenschaftlichen Manuskripts}

Jede wissenschaftliche Arbeit hat eine immer gleiche Struktur:

- 1: Titel

2: Zusammenfassung

3: Einleitung

4: Material und Methoden

- 5: Ergebnisse

6: Diskussion

7: Referenzen

Dabei bilden Einleitung, Methoden, Ergebnisse und Diskussion das Kernstück des Manuskripts und müssen entsprechend ihrer Funktion mit den richtigen Inhalten gefüllt werden. Jeder dieser vier Abschnitte dient zur Beantwortung einer bestimmten Frage, die ausschließlich in diesem Abschnitt beantwortet werden sollte [15]:

- Einleitung $\rightarrow$ Warum wurde die Studie durchgeführt?

- Material und Methoden $\rightarrow$ Wie wurde die Studie durchgeführt?

- Ergebnisse $\rightarrow$ Was wurde bei der Studie herausgefunden?

- Diskussion $\rightarrow$ Welche Bedeutung haben diese Ergebnisse?

\section{Wie beginnen - Was bringt man wann zu Papier?}

Bevor man mit dem Schreiben beginnt, sollte man sich das genaue Ziel der Studie und die definierte Kernfrage wieder vor Augen führen $[4,6,9]$. Dazu gehört eine erneute umfassende Literaturrecherche, bei der alle für die Thematik relevanten Artikel analysiert und ihrer Relevanz sowie Aktualität nach geordnet werden [9]. Dabei sollten die wichtigsten Artikel verinnerlicht und als Wegweiser benutzt werden [6].

Ein Teil des Manuskripts, nämlich die Einleitung und der Methodenteil, kann bereits verfasst werden, bevor die Untersuchungen abgeschlossen sind. In der Regel ist es jedoch so, dass die Studie bereits abgeschlossen wurde, wenn man mit dem Schreiben beginnt. In diesem Fall bietet es sich an, die erhobenen Daten in Form von Tabellen und Abbildungen zusammenfassen. Diese werden in eine Reihenfolge gebracht, die später den roten Faden des Manuskripts ergibt [13]. Sobald man selbst eine Vorstellung von der Struktur hat, ist es Zeit, seinen Mentor zu konsultieren, der in der Regel der Letztautor des Manuskripts und Ideengeber der Untersuchungen ist. Mit diesem werden die zu erwartenden oder bereits erhobenen Ergebnisse analysiert und der Aufbau des Manuskripts festgelegt. Oft kristallisiert sich die Kernaussage einer Arbeit erst heraus, nachdem die Ergebnisse analysiert und übersichtlich zu Papier gebracht wurden [6]. Das wichtigste Ziel dieses Mentorengesprächs ist es, den Fokus des Artikels zu definieren und die Kernfrage zu formulieren [4].

Es empfiehlt sich, zunächst verschiedene vorläufige Titel zu generieren. Dadurch ist man gezwungen, sich erneut über die Kernaussage Gedanken zu machen und dem Manuskript eine Richtung zu geben. Man sollte eine vorläufige Formatierung vornehmen, indem man Überschriften und Zwischenüberschriften einfügt. Dadurch erhält man ein Dokument, in das jederzeit Gedanken, Ideen, Abbildungen und Literaturstellen an der richtigen Stelle einfügt werden könnten [6]. Ist die Studie bereits durchgeführt worden, können die Abbildungen und Tabellen erstellt und jeweils mit einem resümierenden Titel versehen werden. Prinzipiell sollte anhand dieser das gesamte Projekt nachvollziehbar sein. Wenn die Abbildungen und Tabellen für sich eine zusammenhängende Geschichte erzählen und in einer Kernaussage münden, ist es an der Zeit, das Manuskript zu schreiben [13].

Am einfachsten ist es, mit dem Material und Methoden Teil zu beginnen und den Aufbau der Studie wiederzugeben. Anschließend bietet es sich an, den Ergebnissteil zu schreiben, da man den Aufbau der Studie genau vor Augen hat (Material und Methoden) und bereits die Abbildungen vorliegen hat, die den roten Faden vorgeben [13]. Im Anschluss sollte man zusätzlich zu der bereits durchgeführten eine erneute und sorgfältige Literaturrecherche vornehmen, um die Einleitung mit möglichst aktuellem Bezug zu schreiben und damit die Notwendigkeit der eigenen Studie zu untermauern. Am Ende wird die Diskussion geschrieben, welche die eigenen Resultate (Ergebnisteil) in Bezug zu dem derzeitigen Stand der Wissenschaft (Einleitungsteil) setzt. Ist die Untersuchung noch nicht abgeschlossen, kann alternativ als Erstes mit dem Einleitungsteil begonnen werden. Hierfür ist die eingangs genannte intensive Literaturrecherche notwendig, welche dazu beiträgt, die konkrete Fragestellung des Projekts zu hinterfragen und möglicherweise auf einen anderen, eventuell aktuelleren Fokus zu lenken. Die Zusammenfassung des Manuskripts, der „Abstract“, kann ebenfalls zu verschiedenen Zeitpunkten erstellt werden. Möglicherweise sind Teile der Daten bereits auf einem Kongress vorgestellt worden, sodass hierfür schon eine Matrize vorhanden ist. Man kann die Zusammenfassung ganz zu Beginn schreiben, um sich zu fokussieren und einen roten Faden zu finden. Alternativ kann man die Zusammenfassung auch zum Schluss schreiben und die bereits geschriebenen Abschnitte des Hauptdokumentes hierfür entsprechend kondensieren.

\section{Empfehlungen zur Ausgestaltung der Abschnitte $\nabla$}

Im Folgenden wird die Aufgabe und Struktur jedes einzelnen Abschnitts eines wissenschaftlichen radiologischen Manuskriptes erläutert. Eine Übersicht gibt $\bullet$ Tab. 1. Die Reihenfolge der Erläuterungen entspricht der Kapitelfolge im Manuskript und nicht der Reihenfolge, in der diese Kapitel erstellt werden sollten.

\section{Titel}

Die Wahl des Titels kann darüber entscheiden, ob ein Herausgeber das Manuskript als interessant oder als für das Journal nicht adäquat bewertet und ohne weiteren Review-Prozess ablehnt. Weiterhin entscheidet der Titel darüber, wie gut der Artikel später in Suchmaschinen wie PubMed gefunden wird und ob das Interesse potenzieller Leser geweckt wird $[8,18]$. 
Tab. 1 Inhalt und Reihenfolge der einzelnen Kapitel und Unterkapitel eines wissenschaftlichen radiologischen Manuskripts.

1. Titel

2. Zusammenfassung

3. Einleitung

3.1 Relevanz: Aufzeigen der Problemstellung

3.2 Originalität: Notwendigkeit der Studie

3.3 Definierte Fragestellung: Ausdrückliches Ziel der Studie

4. Material und Methoden

4.1 Studiendesign

4.2 Techniken

4.3 Mess- und Auswertemethoden

4.4 Gewährleistung der Datenqualität

4.5 Statistische Analyse

5. Ergebnisse

5.1 Deskriptive Statistik der Studienpopulationen

5.2 Resultate, Validität und Signifikanz der Messungen

6. Diskussion

6.1 Synopsis der wichtigsten Ergebnisse

6.2 Interpretation der eigenen Ergebnisse

6.3 Interpretation im Kontext der bisherigen Datenlage

6.4 Klinische Bedeutung

6.5 Limitationen

6.6 Schlussfolgerung

7. Referenzen

Adaptiert nach M. A. Kliewer.

Der Titel sollte kurz, prägnant und leserlich sein, unverständliche Abkürzungen sollten vermieden werden, wohingegen bekannte Akronyme wie z.B. „MRI“ oder „CT“ erlaubt sind [8, 15, 18]. Gleichzeitig müssen alle relevanten Merkmale der Studie enthalten sein: Patientenpopulation, Erkrankung und Bildgebungstechnik. Zwei Sätze mit einem Doppelpunkt zu verbinden verstärkt gleichzeitig die Aussage und ermöglich ein kurze Formulierung, beispielsweise „Quantitative assessment of bone marrow attenuation values at MDCT: an objective tool for the detection of bone bruise related to occult sacral insufficiency fractures“ [6, 19]. Alternativ kann ein prägnantes, positives Statement des wichtigsten Resultats formuliert werden, wie z.B. „Erfolgreiche durchleuchtungsgesteuerte Anlage einer perkutanen Gastrostomie bei voroperiertem Billroth-II-Magen“ [20]. Solche Formulierungen sind einprägsam und wecken die Aufmerksamkeit.

\section{Zusammenfassung}

Die Zusammenfassung ist ein Kondensat des gesamten Manuskripts. Mehr noch als der Titel ist die Zusammenfassung dafür entscheidend, ob das Manuskript in den BegutachtungsprozessProzess kommt, ob es durch PubMed oder andere Suchmaschinen gefunden wird und ob ein potenzieller Leser sich dazu entscheidet, das Manuskript zu lesen [8]. Daher ist in der Zusammenfassung darauf zu achten, dass sowohl Kernfrage als auch Relevanz der Studie herausgearbeitet werden. In der Regel ist die Zusammenfassung auf 150 bis 250 Wörter limitiert und in Ziel, Methoden, Ergebnisse und Schlussfolgerungen gegliedert.

Zunächst muss das Ziel der Studie in ein oder zwei Sätzen klar dargestellt werden, welches identisch zum Ziel der Einleitung sein muss [4, 9]. Im Methodenteil werden Studienpopulation, Techniken, Variablen, statistische Tests und Art der Studie (prospektiv oder retrospektiv) angegeben. Der Ergebnissteil enthält nur die wichtigsten Ergebnisse unter Angabe der statistischen Signifikanz. Im letzten Abschnitt sollte in ein oder zwei Sätzen eine zusammenfassende Schlussfolgerung bzw. Kernaussage
(Take Home Message) formuliert werden. Die Schlussfolgerung muss das Ziel der Studie reflektieren und aufgrund der im Ergebnisteil dargelegten Daten nachvollziehbar sein.

\section{Einleitung}

Die Einleitung liefert die Begründung für die Durchführung der Untersuchungen. Der Leser soll verstehen, warum er das Manuskript lesen sollte - „Why should I read this paper?“. Im Allgemeinen werden in der Einleitung a) die Relevanz der Thematik, b) die Originalität der Studie sowie c) die Kernfrage der Studie erörtert [6]. Es bietet sich an, zunächst alle Argumente zu sammeln und dann im Rahmen der iterativen Überarbeitungen die wichtigsten zu selektieren und die übrigen herauszustreichen. Eine Einleitung ist keine Diskussion, die die eigenen Untersuchungen bereits bewertend diskutiert.

\subsection{Relevanz: Aufzeigen der Problemstellung}

Der erste Abschnitt beleuchtet das diagnostische oder interventionelle Problem einer bestimmten Erkrankung. Hier wird die konkrete Fragstellung aufgezeigt, verallgemeinernde Aussagen sollten vermieden werden. Der letzte Satz dieses Abschnitts sollte darlegen, mit welchen Verfahren das Problem adressiert wird.

\subsection{Originalität: Notwendigkeit der Studie}

Im zweiten Abschnitt werden bisherige Lösungsansätze geschildert und mit aktuellen Literaturstellen kurz belegt [9]. Die Notwendigkeit zu einer weiteren, nämlich der eigenen Studie auf diesem Gebiet wird deutlich gemacht. Entweder wird auf zu überkommende Schwächen bisheriger Untersuchungen oder bisher noch nicht betrachtete Aspekte hingewiesen. Alternativ ist die eigene Studie eine logische Fortführung von bisher zu diesem Thema durchgeführten Arbeiten. Es muss herausgearbeitet werden, dass die vorliegende Untersuchung den Wissenshorizont erweitert und dass die Fragestellung gelöst werden kann [9]. Man sollte vermeiden, vorherige Studien lediglich mit der Begründung einer größeren Studienpopulation zu bestätigen.

\subsection{Definierte Fragestellung: Ausdrückliches Studienziel}

Hier wird aus dem vorgenannten die übergeordnete Rationale für die eigenen Untersuchungen hergeleitet und eine einzelne Kernfrage oder Hypothese unmissverständlich formuliert [4, 9]. Eine wenig präzise Fragestellung ist einer der häufigsten Ablehnungsründe eines Manuskripts [6, 9]. Der letzte Satz der Einleitung sollte beispielsweise so beginnen: „Das Ziel unserer Untersuchungen war.....

\section{Material und Methoden}

Der Methodenteil ist in mehrere Abschnitte gegliedert und wird zur besseren Übersichtlichkeit durch Zwischenüberschriften gegliedert [6]. Alle Experimente sind eindeutig und nachvollziehbar zu beschreiben: Der Leser muss verstehen, wie die präsentierten Daten erhoben wurden. Anhand des Methodenteils soll die Studie reproduzierbar sein $[9,15]$.

\subsection{Studiendesign}

Zunächst ist anzuführen, ob die Untersuchungen an Menschen entsprechend den ethischen Grundsätzen der Deklaration von Helsinki durchgeführt worden sind, durch einen Ethikantrag genehmigt wurden und ob sich die Patienten mit der Auswertung ihrer Daten schriftlich einverstanden erklärt haben [9]. Bei Tierversuchen ist die Angabe eines genehmigten Tierversuchsantrags obligat. Es folgt die Angabe der Studienart: retrospektiv oder pro- 
spektiv. Handelt es sich um eine retrospektive Studie, werden an dieser Stelle die demografischen Daten der Patienten wie Alter und Geschlecht aufgeführt. Handelt es sich um eine prospektive Studie, sind die demografischen Daten des Kollektivs Teil der Ergebnisse und werden im Ergebnissteil aufgeführt. Die Einschlussund Ausschlusskriterien der Studie müssen explizit genannt werden.

\subsection{Techniken}

Hier wird dargelegt, in welcher Reihenfolge und mit welchen Techniken die Daten erhoben wurden. Dazu gehört bei radiologischen diagnostischen Studien immer der Gerätetyp unter Angabe und Herkunft des Herstellers, die Untersuchungstechnik, ggf. Art und Menge von Kontrastmittel sowie alle weiteren eingesetzten Verfahren. Es müssen alle relevanten technischen Parameter aufgeführt werden. Bei interventionellen therapeutischen Studien müssen alle eingesetzten Instrumente wie z.B. Katheter, Führungsdrähte, Stents oder Embolisationsmaterialien so angegeben werden, dass diese eindeutig identifizierbar sind.

\subsection{Mess- und Auswertemethoden}

In diesem Abschnitt muss klar dargelegt werden, welche Daten wie erhoben wurden. Es müssen für die angewandten Messund Auswertemethoden klare Grenzwerte und Kriterien sowie Ranking-Systeme definiert werden. Beispielsweise wird der ZNS-Befall bei der Multiplen Sklerose nicht in „leicht, mittel und schwer" eingeteilt, sondern z. B. die Anzahl der Läsionen je Kategorie klar festgelegt „0; $1-20 ; 21-50$ und >50“. Je klarer diese Kriterien definiert sind, desto valider, nachvollziehbarer und reproduzierbarer sind die erhobenen Daten.

\subsection{Gewährleistung der Datenqualität}

Dieser Abschnitt entscheidet über die Qualität des gesamten Manuskripts und muss dementsprechend schon bei der Planung der Studie sorgfältig bedacht werden [4]. Wie genau und von wem sind die Daten ausgewertet worden? Dazu gehört die Angabe der Anzahl Auswerter und ob diese „verblindet“ waren sowie ob die Daten im Konsensus oder getrennt durch die Auswerter ausgewertet wurden. Wurde die Intra- und Interobservervariabilität bestimmt, lässt sich die Reliabilität der Studie bzw. die evaluierten Methoden beurteilen. Weiterhin muss ein Referenz-/Goldstandard definiert werden, mit dem die zu überprüfenden Techniken verglichen werden. Das kann z. B. eine andere Bildgebungstechnik, Histologie oder Folgeuntersuchung sein. Auch diese Methoden müssen explizit erläutert werden, wie z.B. das Zeitintervall zur Folgeuntersuchung. Hieran lässt sich die Validität der Untersuchungsmethoden beurteilen. Die Reliabilität und die Validität der erhobenen Daten sind entscheidende Kriterien für die Wertigkeit des gesamten Manuskripts [4].

\subsection{Statistische Analyse}

In diesem Abschnitt werden die statistischen Tests zur Überprüfung der Signifikanz in der Reihenfolge der erhobenen Untersuchungsergebnisse dargelegt [9]. Dadurch kann der Gutachter und Leser die Validität und Signifikanz besser beurteilen. Hierbei ist anzumerken, dass man einen Statistiker grundsätzlich vor Beginn einer Studie hinzuziehen sollte und nicht erst, nachdem die Daten erhoben wurden. Es ist ratsam, einfachere statistische Tests selbst zu erlernen und schwierigere Tests zumindest nachvollziehen zu können. Ersteres spart viel Zeit und Letzteres hilft bei der Planung zukünftiger Studien. Schließen könnte dieser Ab- schnitt z. B. mit dem Satz: „p-Werte $<0,05$ wurden als statistisch signifikant gewertet“.

\section{Ergebnisse}

Der Ergebnissteil sollte sich in der Reihenfolge am Methodenteil orientieren und ebenfalls mit Zwischenüberschriften unterteilt werden. Es sollten nur Daten präsentiert werden, die zur Beantwortung der Kernfrage dienen. Für gewöhnlich werden mehr Daten im Rahmen einer Studie erhoben, als zur Beantwortung der Fragestellung nötig sind. Diese zusätzlichen Daten gehören nicht in das Manuskript. Es die Aufgabe des Autors, und nicht die des Lesers, die wesentlichen Ergebnisse herauszuarbeiten [15]. Die Daten werden im Ergebnissteil nicht kommentieret oder bewertet. Die Interpretation erfolgt ausschließlich in der Diskussion.

\subsection{Deskriptive Statistik der Studienpopulationen}

Handelt es sich um eine prospektive Studie, werden zunächst die demografischen Daten der Patienten und die Merkmale der untersuchten Studienpopulationen präsentiert. Hierfür bietet sich eine Tabelle an.

\subsection{Resultate, Validität und Signifikanz der Messungen}

Hier werden die Resultate der durchgeführten Untersuchungen entsprechend der Reihenfolge des Methodenteils präsentiert. Ausgewählte Untersuchungsbilder einzelner Patienten werden präsentiert, welche die vorgenommenen Messungen, Interventionen oder Kategorisierungen illustrieren. Die Daten aller Patienten werden übersichtlich in tabellarischer oder grafischer Form präsentiert. Die Abbildungen sollten eine hervorragende Qualität aufweisen und mit einer Auflösung von 300 dpi in dem von der Zeitschrift geforderten Format (z. B. JPEG, TIFF) generiert werden [21]. Der Text sollte die zum Ergebnisteil gehörenden Abbildungen erläutern und deren Aussage verstärken, jedoch keinesfalls detailliert deren gesamten Inhalt wiederholen. Widersprüchlichkeiten und Doppeldeutigkeiten müssen vermieden werden. Die präsentierten Zahlen müssen in sich stimmig sein. Ansonsten wird die Qualität der gesamten Studie unmittelbar angezweifelt und überkritisch beurteilt. Messwerte sollten als Mittelwerte unter Angabe der Standardabweichung und der statistischen Signifikanz angegeben werden. Die Angaben müssen so präzise wie möglich sein, schwammige Adjektive wie „die meisten“, „einige“ oder „wenige“ müssen vermieden werden, da sie erstens nicht genau sind und zweitens eine Wertung beinhalten. Neben den eigentlichen Resultaten wird auch die Messung der Reliabilität (Intra- und Interoberserver Variabilität) sowie die Validität der Daten im Vergleich zum Goldstandard präsentiert.

\section{Diskussion}

Der Diskussionsteil ist ein besonders anspruchsvoller Abschnitt des Manuskripts. Wir empfehlen, dem Vorschlag von M. A. Kliewer zu folgen, der den Diskussionsteil in die sechs im Folgenden beschriebenen definierten Abschnitte gliedert [6]. Diese Gliederung sollte man innerlich bereits bei Beginn der Manuskripterstellung durchführen. Dadurch können jederzeit Ideen, Gedanken, Argumente und Referenzen passend eingefügt werden, die den Diskussionsteil entsprechend gestalten. Insbesondere ist darauf zu achten, dass sich der Fokus der Diskussion um die eigenen Ergebnisse dreht und das Thema der Überschrift widerspiegelt. Langatmige Diskussionen anderer Studien oder sogar des gesamten Forschungsfelds müssen tunlichst vermieden werden. Im Diskussionsteil können durchaus neue Hypothesen aufgestellt werden, diese müssen jedoch deutlich als solche gekennzeichnet 
werden, damit diese vom Leser nicht fälschlicherweise als Schlussfolgerungen missverstanden werden.

\subsection{Synopsis der wichtigsten Ergebnisse}

Im ersten Satz sollte nochmals die Fragestellung wiederholt werden [13]. Anschließend sollten die wichtigsten Ergebnisse in wenigen Sätzen zusammengefasst werden. Dabei sollte man sich auf die wesentlichen Kernaussagen beschränken und langatmige Wiederholungen des Ergebnisteils vermeiden [6]. An dieser Stelle werden weder die zugrunde liegende Erkrankung noch das bestehende diagnostische Dilemma erörtert, da dies bereits in der Einleitung erläutert wurde. Schließen sollte man mit der Beantwortung der Kernfrage oder Hypothese: Wurde diese bestätigt oder verworfen?

\subsection{Interpretation der eigenen Ergebnisse}

Der zweite Abschnitt der Diskussion reflektiert die Bedeutung der eigenen Ergebnisse. Diese müssen kritisch hinterfragt und interpretiert werden. Der mit der Studie erzielte wissenschaftliche Fortschritt muss klar formuliert werden [9]. Dabei darf die Bedeutung der eigenen Ergebnisse nicht überbewertet werden. Superlative wie „...wir sind die Ersten...“ etc. sollten vermeiden werden. Wenn man tatsächlich der oder die Erste ist, dann wissen das in der Regel die Gutachter und Leser. Generell sollte von Eigenwerbung abgesehen werden und Begriffe wie „einzigartig“, „hochinnovativ“ und „extrem wertvoll“ vermieden werden [9]. Es dürfen nur Schlussfolgerungen gezogen werden, die tatsächlich durch die eigenen Daten belegt sind.

\subsection{Interpretation im Kontext der bisherigen Datenlage}

Die eigenen Daten werden mit den Ergebnissen bisheriger Studien verglichen, deren Daten konkret referiert werden sollten [9]. Die Bedeutung von Übereinstimmungen oder Diskrepanzen ist zu hinterfragen und zu diskutieren. Insbesondere muss Stellung zu den Studien genommen werden, die im Einleitungsteil zitiert wurden. Man sollte sich nicht scheuen, auch widersprüchliche Ergebnisse hervorzuheben und die möglichen Gründe zu diskutieren. Möglicherweise sind gerade diese die interessantesten Ergebnisse [9].

\subsection{Bedeutung}

In diesem Abschnitt wird erläutert, ob und wie genau die Ergebnisse der Studie den klinischen Alltag und die Patientenversorgung beeinflussen oder experimentelle Ergebnisse neuen Erkenntnisgewinn bedingen [9]. Wenn durch die Studie ein Verfahren in der eigenen Abteilung umgestellt wurde, sollte dies hier dokumentiert werden.

\subsection{Limitationen}

Jede Studie hat Limitationen, die in der Diskussion eines jeden Manuskripts behandelt werden müssen. Mutmaßlich sind dem Gutachter („expert in the field“) die Limitationen bewusst, dem „gewöhnlichen“ Leser jedoch nicht. Nennung und Diskussion der Limitationen zeigt, dass man aufgeschlossen, objektiv und unbefangen ist [9]. Es zeigt weiterhin, dass die gezogenen Schlussfolgerungen vor diesem Hintergrund gemacht wurden und daher als valide $\mathrm{zu}$ interpretieren sind.

\subsection{Schlussfolgerung}

Im letzten Abschnitt der Diskussion sollte man die wichtigsten Erkenntnisse zusammenfassen. In einem Satz wird eine umfassende Schlussfolgerung gezogen. Diese „Take Home Message“ spiegelt die des Abstracts wider. Haben sich neue Fragestellun- gen ergeben, kann ein Ausblick auf zukünftige Studien gegeben werden.

\section{Referenzen}

Bei Originalarbeiten sollte man sich auf etwa 30 Referenzen beschränken und diese entsprechend des Journals formatieren. Es sollten möglichst aktuelle und hochrangige Publikationen zitiert werden. Hierdurch wird die Aktualität und Relevanz der Fragestellung sichergestellt $[9,17]$.

\section{Die finale Version \\ $\nabla$}

Der Erstautor beginnt mit der Erstellung des Manuskripts. Überarbeitungen erfolgen in mehrfachen Iterationen mit allen weiteren Autoren. Man sollte alle Korrekturvorschläge aus der Arbeitsgruppe sorgfältig bedenken. Unstimmigkeiten oder Verständnisschwierigkeiten müssen sorgfältig bearbeitet werden: Wenn es ein CoAutor nicht versteht, wird es der Gutachter ebenfalls nicht verstehen, und daher müssen die betreffenden Passagen des Dokuments klarer formuliert werden. Lange und unverständliche Sätze müssen gekürzt und vereinfacht werden.

Zu diesem Zeitpunkt sollten alle Zahlen im Ergebnisteil nochmals kontrolliert werden. Ist das Manuskript für ein Journal in englischer Sprache bestimmt, sollte das Manuskript zusätzlich von einem „native speaker“ korrigiert werden. Insbesondere in dieser Phase erfolgt durch alle Autoren eine Kürzung und Präzisierung des Manuskripts. Durch die wiederholten Überarbeitungen verbessert sich die Lesbarkeit und Verständlichkeit des Manuskripts [15]. Alle Autoren müssen einen relevanten Beitrag zu der Arbeit geleistet haben und das Manuskript gelesen haben.

\section{Auswahl des Journals \\ $\nabla$}

Die Auswahl des Journals sollte nach Rücksprache mit einem erfahrenen Kollegen erfolgen. Dieser kann die Relevanz und den Wert der Studie besser beurteilen und ein geeignetes Journal empfehlen. Hierbei orientiert man sich an der Zielgruppe, dem Interessengebiet des Journals und ggf. dem Impact Factor. Ist die Untersuchung für eine spezialisierte Expertengruppe, die allgemeine radiologische oder sogar generell medizinische Leserschaft von Interesse? Hat man sich für ein Journal entschieden, muss das Manuskript genau den Vorgaben entsprechend formatiert werden. Neben den radiologischen Fachzeitschriften kommen in bestimmten Fällen auch Journale aus anderen Fachgebieten für die Publikation infrage. In diesem Falle müssen bestimmte Formulierungen der Vorbildung der Leserschaft anpasst werden. Die richtige Wahl des Journals kann die Wahrscheinlichkeit einer Ablehnung deutlich reduzieren. Allerdings sollte man sich dessen bewusst sein, dass der überwiegende Anteil aller eingereichten Manuskripte bei der ersten Einreichung abgelehnt werden, am Ende jedoch über $80 \%$ aller Artikel publiziert werden [3].

\section{Einreichung des Manuskripts \\ $\nabla$}

Bevor man das Manuskript auf dem Internetportal des Journals hochlädt, sollte man sich nochmals vergewissern, dass das Manuskript genau den spezifischen Vorgaben entspricht. Dazu gehört die richtige Gliederung, Einhaltung des Limits für die Länge des Manuskripts, die Anzahl der Abbildungen, Tabellen und Refe- 
renzen [15]. Weiterhin sollte man überprüfen ob alle Zahlen stimmen, die Referenzen richtig zugeordnet sind und nochmals eine Rechtschreibkorrektur durchführen. Darüber hinaus sollte man kontrollieren, ob die Abbildungen im richtigen Dateiformat vorliegen und die Referenzen nach dem Stil des Journals formatiert sind. Alle Abbildungen und Tabellen müssen im Manuskript erwähnt und richtig zugeordnet sein sowie eine entsprechende Legende aufweisen. Weiterhin ist darauf zu achten, ob das betreffende Journal ein „double-blinded Peer Review“ durchführt. Dann ist das Manuskript zu anonymisieren, indem die Autoren und Institutsnamen aus dem Hauptdokument entfernt werden und Formulierungen wie „.... wie wir in der Vergangenheit zeigen konnten (Eigenzitat)." vermieden werden.

Hält man sich nicht an die Vorgaben, wird das Manuskript von der Redaktion mit der Aufforderung zur Überarbeitung zurückgeschickt, was mit einer nicht unbedeutenden Zeitverzögerung verbunden sein kann. Ferner sollte man einen Brief an den Herausgeber vorbereitet haben, in welchem knapp die Gründe für die Publikation in dem betreffenden Journal dargelegt werden. In vielen Fällen fordern die Journale, neben „Keywords“ zusätzlich drei bis fünf sogenannte „Keypoints“, die man sorgfältig formuliert haben sollte. Diese Keypoints sollten bereits im Vorfeld zusammen mit dem übrigen Manuskript wiederholt überarbeitet werden, da diese neben der Überschrift und dem Abstract gesondert hochgeladen und als Erstes von den Editoren begutachtet werden.

\section{Schlussfolgerungen}

$\nabla$

Eine unklare Fragestellung oder ein wandernder Fokus sind der häufigste Grund für die Ablehnung eines wissenschaftlichen Manuskripts. Man sollte also eine dedizierte Kernfrage bzw. einen speziellen Fokus des Manuskripts definieren und darauf das gesamte Manuskript konsequent ausrichten. Entlang dieses roten Fadens wird die eingangs definierte Kernfrage oder aufgestellte Hypothese beantwortet bzw. überprüft. Neben der konsequenten Verfolgung des roten Fadens sind der richtige Aufbau und die sorgfältige Präparation des Manuskripts von elementarer Bedeutung. Diese kann im Zweifelsfall über die Ablehnung oder Annahme entscheiden.

Zusammenfassend lässt sich sagen, dass ein gut geschriebenes Manuskript so knapp wie möglich, aber auch so präzise wie möglich eine genau definierte Kernfrage beantwortet. Insbesondere für Anfänger ist es nicht einfach, komplizierte Zusammenhänge verständlich darzustellen und die einzelnen Bestandteile eines Manuskripts in die richtige Reihenfolge zu bringen. Die vorliegende Übersicht soll als Vorlage für die richtige Strukturierung eines wissenschaftlichen Manuskripts dienen und Hinweise für eine erfolgreiche Publikation geben. In weiteren Beiträgen werden wir wichtige Eckpunkte zur Planung und Durchführung klinischer und experimenteller Untersuchungen vorstellen.

\section{Danksagungen}

Die strukturierte Unterteilung der einzelnen Unterkapitel erfolgte in Anlehnung an den Leitfaden von M. A. Kliewer [6]. P. B. dankt seinen Mentoren Prof. Friedrich Koch-Nolte und Prof. Gunnar Lund. Wir danken cand. med. Jenny Krause für die sorgfältige Überarbeitung des Manuskripts und die hilfreichen Ratschläge aus der Sicht einer jungen Wissenschaftlerin.

\section{Literatur}

1 Albarran JW, Scholes J. How to get published: seven easy steps. Nurs Crit Care 2005; 10: $72-77$

2 Berk RN. Preparation of manuscripts for radiology journals: advice to first-time authors. Am J Roentgenol 1992; 158: 203-208

3 Chew FS. Fate of manuscripts rejected for publication in the Am J Roentgenol. Am J Roentgenol 1991; 156: 627-632

4 Davidson AJ, Carlin JB. What a reviewer wants. Paediatr Anaesth 2008; 18: $1149-1156$

5 Eyler WR. Uniform requirements for manuscripts submitted to biomedical journals. Radiology 1980; 135: 239-243

6 Kliewer MA. Writing it up: a step-by-step guide to publication for beginning investigators. Am J Roentgenol 2005; 185: 591 - 596

7 Laniado $M$. How to present research data consistently in a scientific paper. Eur Radiol 1996; 6: S16-S18

8 Rosenfeldt FL, Dowling JT, Pepe S et al. How to write a paper for publication. Heart Lung Circ 2000; 9: $82-87$

9 Sheiman R. Writing an Original Research Manuscript: The Do's and Don'ts. Chicago: RSNA; 2009

10 Siegelman SS. Advice to authors. Radiology 1988; 166: 278-280

11 Weber EJ, Callaham ML, Wears RL et al. Unpublished research from a medical specialty meeting: why investigators fail to publish. JAMA 1998; 280: 257-259

12 Welch $H G$. Preparing manuscripts for submission to medical journals: the paper trail. Eff Clin Pract 1999; 2: 131 - 137

13 Wells WA. Me write pretty one day: how to write a good scientific paper. J Cell Biol 2004; 165: 757-758

14 Ziman J. Public knowledge: The social dimension of science: Cambridge University Press.1968

15 Whimster WF. Biomedical Research: How to plan, publish and present it. 2nd ed. London: Springer; 1997

16 Heindel $W$, Schwindt $W$. RöFo: prospects and challenges for the future. Fortschr Röntgenstr 2012; 184: 877-882

17 Bordage G. Reasons reviewers reject and accept manuscripts: the strengths and weaknesses in medical education reports. Acad Med 2001; 76: 889-896

18 Jacques TS, Sebire NJ. The impact of article titles on citation hits: an analysis of general and specialist medical journals. JRSM Short Rep 2010; $1: 2$

19 Henes FO, Groth M, Bley TA et al. Quantitative assessment of bone marrow attenuation values at MDCT: an objective tool for the detection of bone bruise related to occult sacral insufficiency fractures. Eur Radiol 2012; 22: 2229-2236

20 Bannas P, Adam G, Habermann C. Successful image guided implantation of a percutaneous gastrostomy in a previously operated Billroth II stomach. Fortschr Röntgenstr 2009; 181: $904-905$

21 Briscoe MH. Preparing Scientific Illustrations. 2nd ed. New York: Springer; 1995 